\title{
Entropic Ratchet transport of interacting active Browanian particles
}

\author{
Bao-quan Ai ${ }^{1} *$ Ya-feng $\mathrm{He}^{2}$, and Wei-rong Zhong ${ }^{3}$ \\ ${ }^{1}$ Laboratory of Quantum Engineering and Quantum Materials, \\ School of Physics and Telecommunication Engineering, \\ South China Normal University, 510006 Guangzhou, China \\ ${ }^{2}$ College of Physics Science and Technology, \\ Hebei University, 071002 Baoding, China. \\ ${ }^{3}$ Department of Physics and Siyuan Laboratory, \\ College of Science and Engineering, \\ Jinan University, 510632 Guangzhou, China.
}

(Dated: November 7, 2018)

\begin{abstract}
Directed transport of interacting active (self-propelled)Brownian particles is numerically investigated in confined geometries (entropic barriers). The self-propelled velocity can break thermodynamical equilibrium and induce the directed transport. It is found that the interaction between active particles can greatly affect the ratchet transport. For attractive particles, on increasing the interaction strength, the average velocity firstly decreases to its minima, then increases, and finally decreases to zero. For repulsive particles, when the interaction is very weak, there exists a critical interaction at which the average velocity is minimal, nearly tends to zero, however, for the strong interaction, the average velocity is independent of the interaction.
\end{abstract}

PACS numbers: 05. 40. -a, 05. 60. Cd, 82. 70. Dd

Keywords: interacting self-propelled particles, confined geometries

*Email: aibq@hotmail.com 


\section{INTRODUCTION}

Diffusion in confined geometries is ubiquitous in nature. The reduction of the coordinates in confined structures can cause the appearance of remarkable entropic effects. More recently, physicists have started to study entropic effects in out-of-equilibrium phenomena such as transport of particles in corrugated channels[1]. Based on the geometry of the channel wall, corrugated channels fall into two categories: Compartmentalized channe [2-4] and smoothly corrugated channels [5-11]. The relevance of entropic barriers to promote entropic transport in confined environments has been recognized in a variety of situations that include molecular transport in zeolites, ionic channels, or in microfluidic devices. The entropic transport in these systems yields important and exhibits peculiar properties.

In previous works, the entropic transport mainly focused on passive particles and few works on the entropic transport have involved active Brownian particles. However, active matters in biological and physical systems have been studied theoretically and experimentally [12 28]. The kinetics of active particles moving in periodic structures could exhibit peculiar behaviors. Active particles or agents are assumed to have an internal propulsion mechanism, which may use energy from an external source and transform it under nonequilibrium conditions into directed motion. Therefore, it would be significant important to study transport behaviors of active Brownian particles in entropic potentials. Recently, Ghosh and coworkers [27] studied the transport of self-propelled particles in periodic entropic potentials and found that ratcheting current can be orders of magnitude stronger than for ordinary thermal potential ratchets. Then, they found that elliptic Janus particles along narrow two-dimensional channels can show giant absolute negative mobility [29] and the mean exit time of Janus particles in two dimensional cavities is very sensitive to the cavity geometry, particle shape, and self-propulsion strength [30]. Yariv and coworkers [31] studied the transport of Brownian swimmers in a periodically corrugated channel by using the reduced Fokker-Planck approach.

In this paper, we mainly studied the ratchet transport of interacting self-propelled particles in periodic entropic potentials. We focus on finding how the interaction between active particles affects the entropic transport. From numerical simulations, it is found that upon variation of the interaction strength, the average velocity exhibits nonmonotonical behaviors. For the attractive case, the average velocity firstly decreases, then increases, and 
finally decreases to zero. For the repulsive case, the interaction affects the transport only for very small interaction strength, for the large repulsive strength, the average velocity is independent of the interaction and tends to that in single-particle system. In the regime of small interaction strength, there exists a critical value of the repulsive strength at which the average velocity takes its minimal value, nearly tends to zero.

\section{MODEL AND METHODS}

In this paper, we consider a set of $N$ interacting self-propelled particles in a periodic twodimensional channel. A self-propelled particle is viewed as characterized by a unit vector $\vec{n}_{i} \equiv\left(\cos \theta_{i}, \sin \theta_{i}\right)$ in the $x y$ plane, defining the direction of the self-propelled velocity. The particles are subjected to both translational and rotational diffusion, with coefficients $D_{0}$ and $D_{\theta}$, respectively. The dynamics of the particle $i$ is described by the following overdamped Langevin equations.

$$
\begin{gathered}
\frac{d \vec{r}_{i}}{d t}=v_{0} \vec{n}_{i}-\mu \sum_{j \neq i} \frac{\partial}{\partial \vec{r}_{i}} V\left(\vec{r}_{i}-\vec{r}_{j}\right)+\sqrt{2 D_{0}} \vec{\xi}_{i}(t), \\
\frac{d \theta_{i}}{d t}=\sqrt{2 D_{\theta}} \xi_{i}^{\theta}(t),
\end{gathered}
$$

where $\mu$ is the mobility and $v_{0}$ is the magnitude of the self-propelled velocity. Gaussian white noise terms for both the translational and rotational motion are characterized by $\left\langle\vec{\xi}_{i}(t)\right\rangle=0$, $\left\langle\xi_{i}^{\alpha}(t) \xi_{j}^{\beta}(s)\right\rangle=\delta_{i j} \delta_{\alpha \beta} \delta(t-s)$ and $\left\langle\xi_{i}^{\theta}(t)\right\rangle=0,\left\langle\xi_{i}^{\theta}(t) \xi_{j}^{\theta}(s)\right\rangle=\delta_{i j} \delta(t-s)$, respectively. Here $i, j=1, \ldots, N$ label the particles and $\alpha, \beta=x, y$ the coordinates of the space. The symbol $\langle\ldots\rangle$ denotes an ensemble average over the distribution of the random forces. $\delta$ is the Dirac delta function. For the sake of simplicity, we have ignored hydrodynamic effects.

The shape of the channel can be described by its radius $\omega(x)$ shown in Fig. 1

$$
\omega(x)=a\left[\sin \left(\frac{2 \pi x}{L}\right)+\frac{\Delta}{4} \sin \left(\frac{4 \pi x}{L}\right)\right]+b,
$$

where $\Delta$ is the asymmetry parameter of the channel shape and $a$ is the parameter that controls the slope of the channel. The radius at the bottleneck is determined by the parameters $a, b$, and $\Delta$.

As for the pair interaction potential $V$, we consider two cases: (A) the attractive potential and $(\mathrm{B})$ the repulsive potential. For case A

$$
V(r)=\frac{1}{2} k_{a} r^{2}
$$




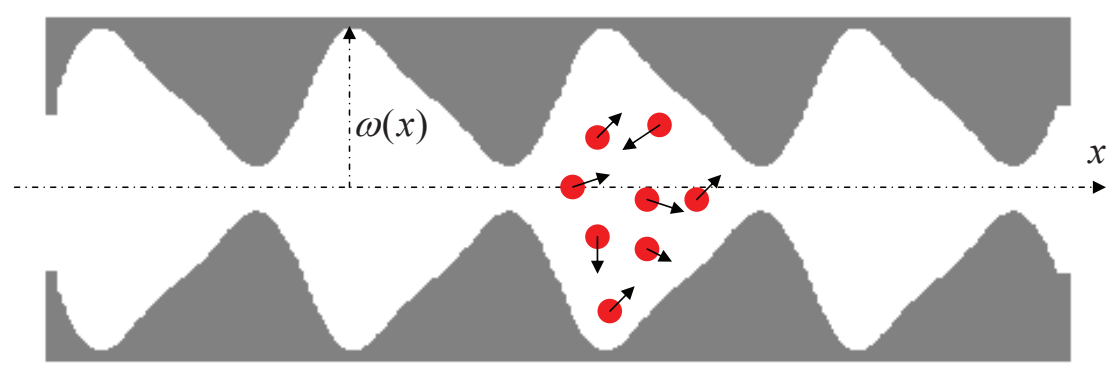

FIG. 1: (Color online) Scheme of the entropic ratchet device: interacting self-propelled particles moving in a two-dimensional channel. The shape is described by the radius $\omega(x)$ of the channel.

and for case $\mathrm{B}$

$$
V(r)=\frac{k_{r}}{r}
$$

where $r$ is the center to center distance between any two particles. $k_{a}$ and $k_{r}$ are the attractive and repulsive strength, respectively.

Rectification of Brownian particles has been the focus of a concerted effort, both conceptual and technological, aimed at establishing net particle transport on a periodic substrate in the absence of external biases. The most important quantity characterizing the rectification in our system is its directional velocity along $x$ direction. Since the Fick-Jacobs equation corresponding to the Langevin equations (11) and (2) can not be solved analytically, we have numerically simulated the overdamped two dimensional dynamics of Brownian particles (1,2) along with the boundary conditions Eq. (3) using an improved Euler algorithm. In the asymptotic long-time regime, the average velocity of particle $i$ along $x$ direction can be obtained from the following formula

$$
v_{i}^{\theta_{0}}=\lim _{t \rightarrow \infty} \frac{\left\langle x_{i}(t)\right\rangle_{\theta_{0}}}{t}
$$

where $\theta_{0}$ is the initial angle of the trajectory. The average velocity after a second average over all $\theta_{0}$ is

$$
v_{i}=\frac{1}{2 \pi} \int_{0}^{2 \pi} d \theta_{0} v_{i}^{\theta_{0}}
$$

The full average velocity is $\bar{v}=\frac{\sum_{i=1}^{N} v_{i}}{N}$. For the convenience of discussion, we define the scaled average velocity $v_{s}=\bar{v} / v_{0}$ through the paper. 


\section{RESULTS AND DISCUSSION}

In our simulations, the integration step time $\Delta t$ was chosen to be smaller than $10^{-4}$ and the total integration time was more than $3 \times 10^{5}$. The stochastic averages reported above were obtained as ensemble averages over $3 \times 10^{4}$ trajectories with random initial conditions. Unless otherwise noted, our simulations are under the parameter sets: $a=\frac{1}{2 \pi}, b=\frac{1.2}{2 \pi}$, $\Delta=1.0$, and $N=4$. The simulation results are reported in Figs. 2-6.

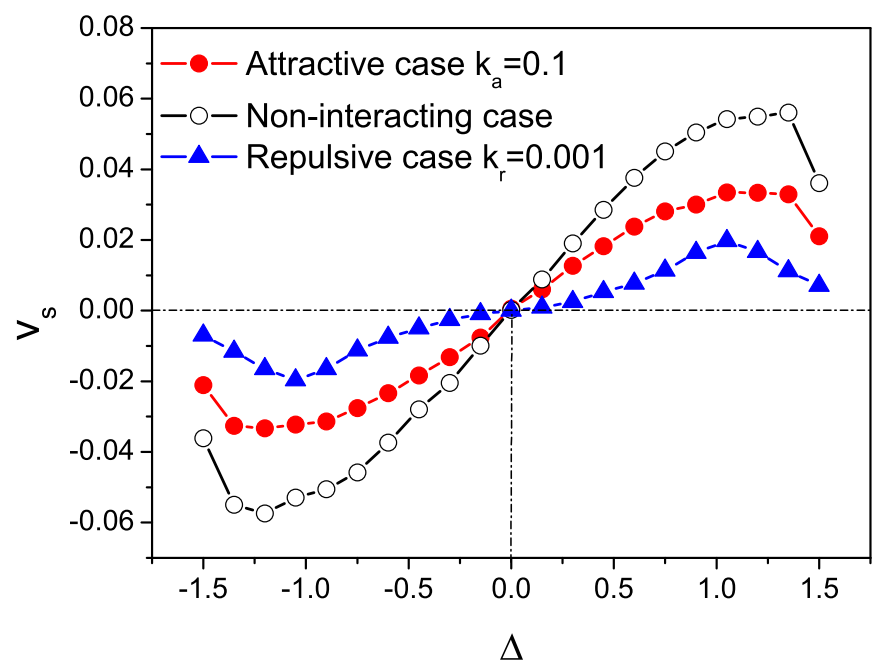

FIG. 2: (Color online) Average velocity $v_{s}$ versus the asymmetric parameter $\Delta$ for three cases. The other parameters are $v_{0}=5.0, k_{a}=0.1, k_{r}=0.001, D_{0}=0.1$, and $D_{\theta}=0.1$.

In Figure 2, we plot the average velocity $v_{s}$ as a function of the asymmetric parameter $\Delta$ for three cases. It is found that the direction of the transport is completely determined by the symmetry of the channel. The average velocity is positive for $\Delta>0$, zero at $\Delta=0$, and negative for $\Delta<0$. When $|\Delta| \rightarrow 0$, the channel is symmetric, so there is no net current. When $|\Delta|>\Delta_{c}$, the channel is blocked, no particles can pass across the cell of the channel. Therefore, there exists an optimal value of $|\Delta|$ at which the average velocity takes its maximal value.

Figure 3(a) shows the average velocity $v_{s}$ as a function of the attractive strength $k_{a}$ for different values of $v_{0}$. On increasing $k_{a}$ from zero, the average velocity $v_{s}$ firstly decreases to its minimal value, then increases, and finally decreases to zero. There exist a valley and 

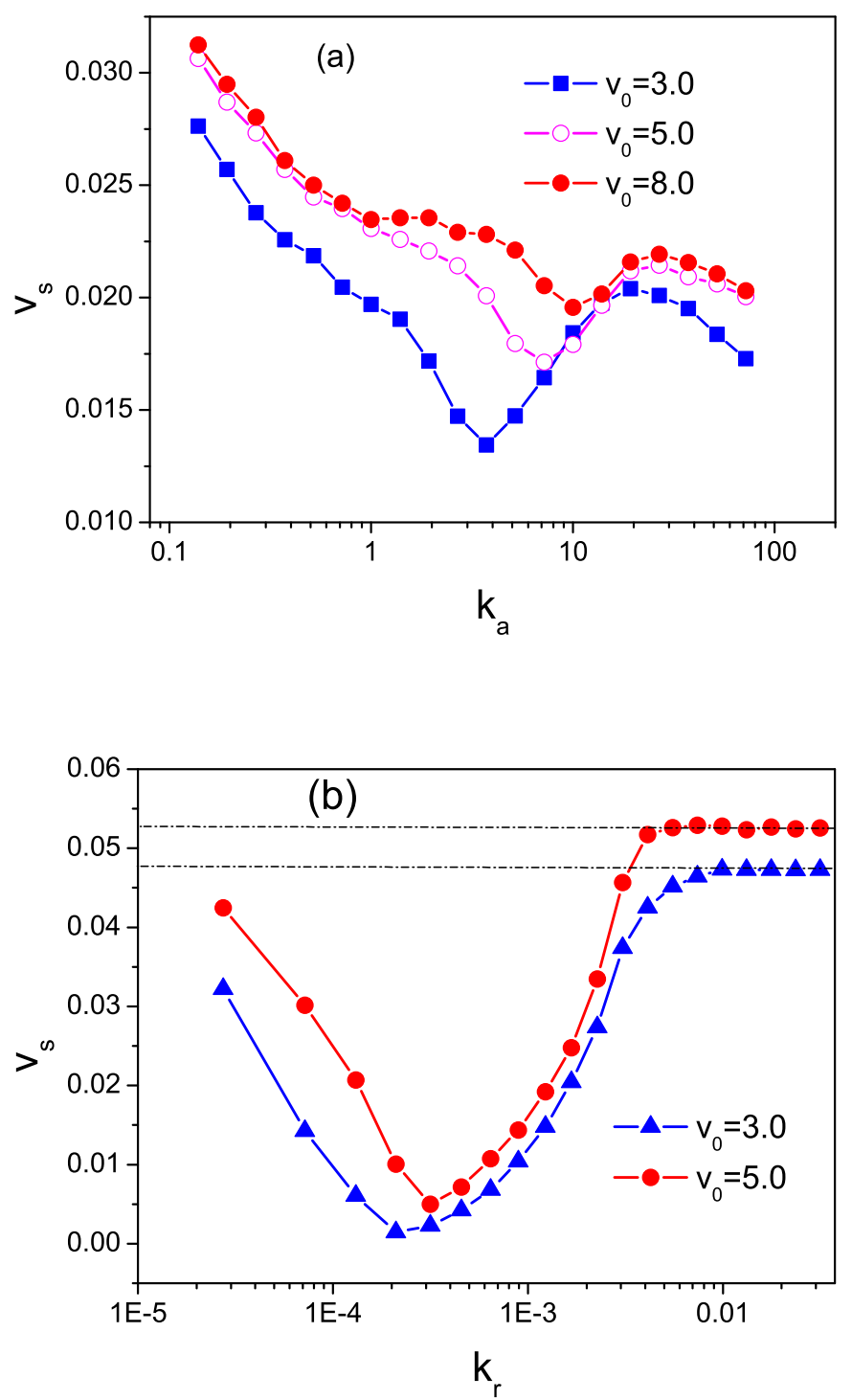

FIG. 3: (Color online) Average velocity $v_{s}$ versus the strength of the interaction potential. (a) For attractive particles $\left(k_{a}\right)$. (b) For repulsive particles $\left(k_{r}\right)$. The other parameters are $v_{0}=5.0$, $D_{\theta}=0.1$, and $D_{0}=0.1$

a peak in the curve and the average velocity takes its minimal value when $k_{a} \simeq v_{0}$. This features can be explained by the following considerations. The attractive interaction in the system can cause two results: (A) reducing the self-propelled driving, which blocks the ratchet transport and (B) activating motion in analogy with thermal noise activated motion for a single stochastically driven ratchet, which facilitates the ratchet transport. When 
$k_{a} \rightarrow 0$, the average velocity attains a constant value (average velocity in noninteracting case). When $k_{a}<v_{0}$, the factor $\mathrm{A}$ dominates the transport, so the average velocity $v_{s}$ decreases when $k_{a}$ increases. When $k_{a}>v_{0}$, the factor $\mathrm{B}$ gradually becomes significant and the average velocity increases with $k_{a}$. However, for very large values of $k_{a}\left(k_{a} \rightarrow \infty\right)$ all particles are gathered as a single particle, both many-body effects and the individual self-propelled driving can be neglected, so the average velocity tends to zero. The average velocity of interacting attractive particles is always smaller than that for the noninteracting case, which indicates that the attractive interaction always blocks the rectification. However, for passive Brownian particles, there exist some values of the attractive strength where the interaction can facilitate the rectification [32].

Figure 3 (b) describes the average velocity $v_{s}$ as a function of the repulsive strength $k_{r}$ for different values of $v_{0}$. It is found that on increasing $k_{r}$, the average velocity $v_{s}$ first decreases to nearly zero value, then increases, and finally tends to a constant. The repulsive interaction in the system can also cause two results: (A) reducing the self-propelled driving (blocking the ratchet transport) and (B) dispersing Brownian particles (facilitating the directed transport). When $k_{r}$ is very small, the factor A dominates the transport and the dispersing effect can be neglected. Therefore, the average velocity $v_{s}$ decreases when $k_{r}$ increases from zero. However, when $k_{r}$ becomes large, the dispersing effect becomes significant, so the average velocity $v_{s}$ increases. For large values of $k_{r}$, the dispersing effect completely dominates the transport and the interaction between Brownian particles can be ignored. When $k_{r} \rightarrow 0$ or $k_{r} \rightarrow \infty$, the average velocity $v_{s}$ tends to the average velocity in the single-particle system, therefore, there exits a critical value of $k_{r}$ (very small value) at which the average velocity $v_{s}$ is minimal, and nearly tends to zero. The critical value of $k_{r}$ depends on the parameters of the system.

Figure 4 shows the dependence of the average velocity $v_{s}$ on the particle number $N$ for both attractive and repulsive cases. For the attractive case, the average velocity $v_{s}$ decreases monotonically with increasing $N$. The average velocity will tend to zero when $N \rightarrow \infty$. The is because the effective attractive driving for large number $N$ can be neglected after the average. For the repulsive case, the average velocity $v_{s}$ is independent of the particle number $N$ when $k_{r}>0.01$. In the infinitely long channel, the repulsive forces between particles disperse particles, the distance between particles become longer and the repulsive forces gradually disappear. 


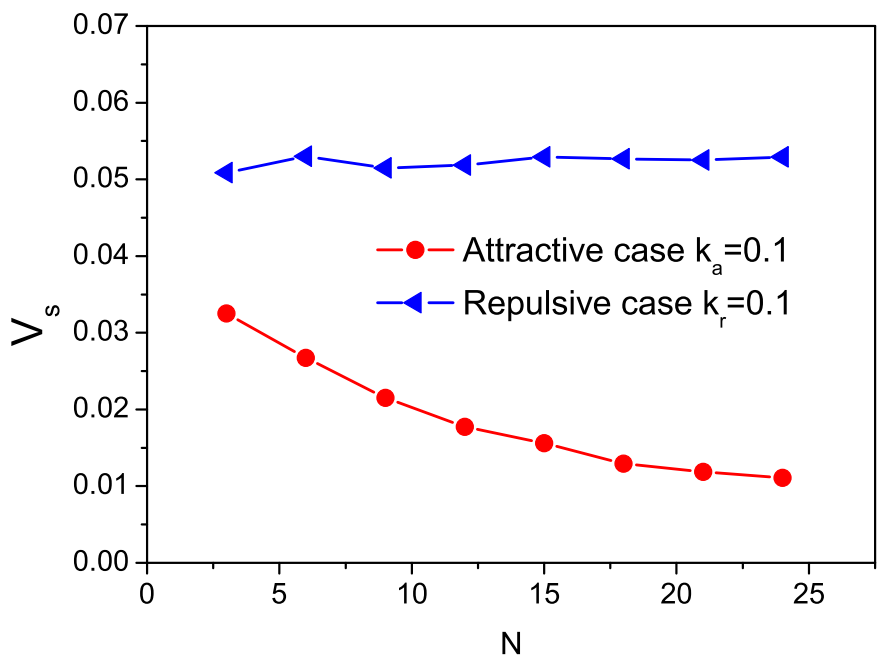

FIG. 4: (Color online) Average velocity $v_{s}$ versus particle number $N$ for both attractive and repulsive particles. The other parameters are $v_{0}=5.0, D_{\theta}=0.1, k_{a}=0.1, k_{r}=0.1$, and $D_{0}=0.1$

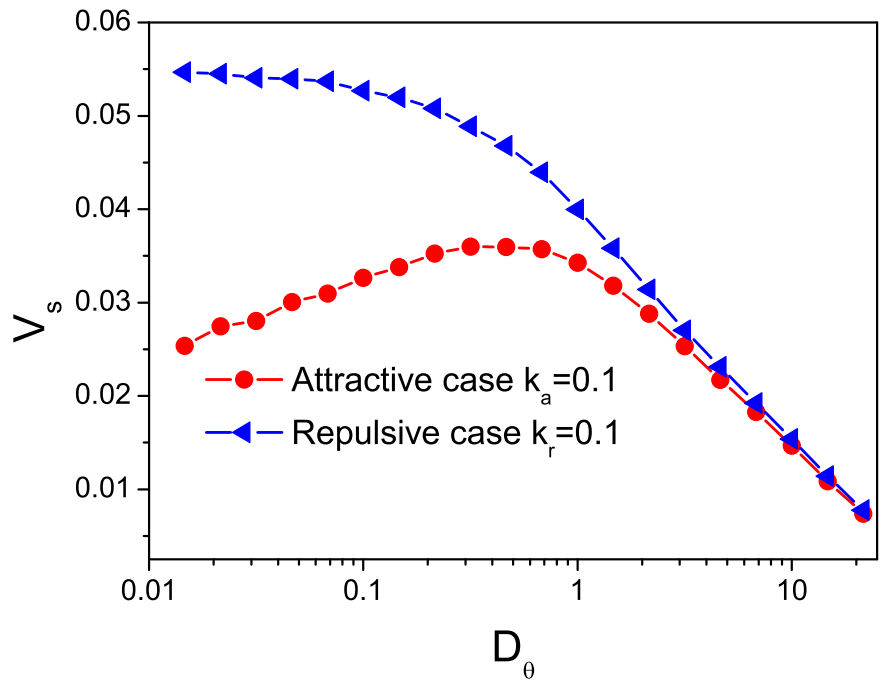

FIG. 5: (Color online) Average velocity $v_{s}$ versus the rotational diffusion $D_{\theta}$ for both attractive and repulsive particles. The other parameters are $v_{0}=5.0, k_{a}=0.1, k_{r}=0.1$, and $D_{0}=0.1$. 
In Fig. 5 , we explore the average velocity $v_{s}$ as a function of the rotational diffusion $D_{\theta}$ for both attractive and repulsive cases. When $D_{\theta} \rightarrow \infty$, transport behaviors are similar for two cases. The self-propelled velocity changes its direction very fast. The self-propelled velocity acts as a zero mean white noise and the nonequilibrium driving in the system disappears, so no directed transport occurs and the average velocity tends to zero. For small values of $D_{\theta}$, the transport behaviors are different for two cases: (A) For the repulsive case $\left(k_{r}=0.1\right)$, the average velocity decreases monotonically when $D_{\theta}$ increases. Especially, when $D_{\theta} \rightarrow 0$, the average velocity tends to a saturate value. In the adiabatic limit, the repulsive force can be expressed by two opposite static force $v_{0}$ and $-v_{0}$, yielding the mean zero velocity $v_{s}=\left[v_{s}\left(v_{0}\right)+v_{s}\left(-v_{0}\right)\right] / 2$, which is similar to the single-particle thermal ratchet [33]. (B) For the attractive case, there exists an optimal value of $D_{\theta}$ at which the average velocity $v_{s}$ is maximal. The increase of $D_{\theta}$ in this case can reduce the self-propelled driving(blocking the ratchet transport) and activate the attractive Brownian particles(facilitating the directed transport). When $D_{\theta}$ increases from zero, the latter factor dominates the transport and the average velocity $v_{s}$ increases to its maximal value. For further increasing $D_{\theta}$, the former factor takes effect, the average velocity $v_{s}$ decreases.

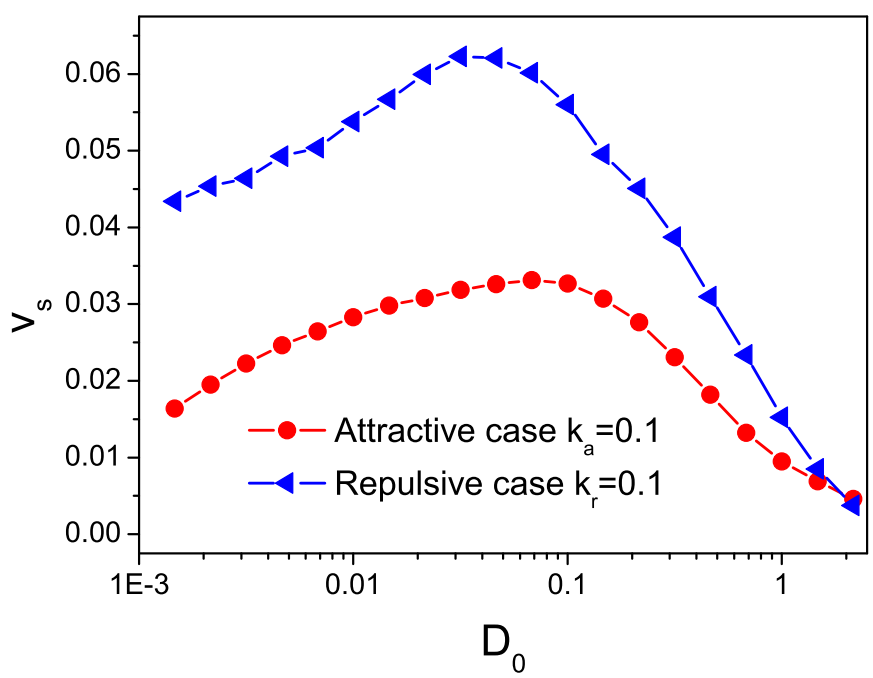

FIG. 6: (Color online) Average velocity $v_{s}$ versus the translational diffusion $D_{0}$ for both attractive and repulsive particles. The other parameters are $v_{0}=5.0, k_{a}=k_{r}=0.1$, and $D_{\theta}=0.1$.

Figure 6 illustrates the impact of the translational diffusion $D_{0}$ on the average velocity $v_{s}$ 
for both the attractive and repulsive cases. From the figure, we can see that the curves are similar for two cases. When $D_{0} \rightarrow 0$, the particles will stay at the bottom of the channel and cannot pass the entropic barrier, so the average velocity $v_{s}$ goes to zero. When $D_{0} \rightarrow \infty$, the translational diffusion is very large, the effect of the asymmetric entropic barrier disappears and the average velocity $v_{s}$ tends to zero. Therefore, there exists an optimal $D_{0}$ value where the average velocity $v_{s}$ is maximal.

\section{CONCLUDING REMARKS}

In this paper, we numerically studied the directed transport of interacting self-propelled Brownian particles in a two-dimensional periodic channel. It is found that the self-propelled velocity acts as the nonequilibrium driving, which can break the themodynamical equilibrium and induce the directed transport. The direction of the transport is completely determined by the symmetry of the channel shape. The interaction between Brownian particles can significantly affect the directed transport. For the attractive case: (1) on increasing the strength $k_{a}$ from zero, the average velocity $v_{s}$ first decreases to its minimal value, then increases, and finally decreases to zero; (2) the average velocity $v_{s}$ decreases monotonically with increase of the particle number $N$; (3) there exists an optimal value of $D_{\theta}$ at which the average velocity $v_{s}$ takes is maximal value, which is different from the noninteracting case, where the average velocity decreases monotonically with increase of $k_{r}$. For the repulsive case: (1) the interaction affects the transport only for very small interaction strength, for the large repulsive strength, the average velocity is independent of the interaction and tends to that in single-particle system. In the regime of small interaction strength, there exists a critical value of the repulsive strength at which the average velocity takes its minimal value, nearly tends to zero; (2) the average velocity $v_{s}$ is independent of the particle number $N$ when $k_{r}>0.01 ;(3)$ the average velocity $v_{s}$ decreases monotonically with increase of the rotational diffusion $D_{\theta}$, especially, it tends to a saturate value when $D_{\theta} \rightarrow 0$. In addition, the average velocity of interacting active Brownian particles is always less than that in the noninteracting case (which indicates the interaction always cannot facilitate the rectification), which is different from the passive case, where the interaction may facilitate the rectification.

This work was supported in part by the National Natural Science Foundation of China 
(Grant Nos. 11175067, 11004082, and 11205044), the PCSIRT (Grant No. IRT1243), the Natural Science Foundation of Guangdong Province, China (Grant No. S2011010003323).

[1] P. Hänggi and F. Marchesoni, Rev. Mod. Phys. 81, 387 (2009); P. Reimann, Phys. Rep. 361, 57 (2002).

[2] M. Borromeo, F. Marchesoni, and P. K. Ghosh, J. Chem. Phys. 134, 051101 (2011); F. Marchesoni, J. Chem. Phys. 132, 166101 (2010); M. Borromeo and F. Marchesoni, Chem. Phys. 375, $536(2010)$.

[3] Yu. A. Makhnovskii, A. M. Berezhkovskii, and V.Yu. Zitserman, J. Chem. Phys. 131, 104705 (2009); A. M. Berezhkovskii, L. Dagdug, Yu. A. Makhnovskii, and V. Yu. Zitserman, J. Chem. Phys. 132, 221104 (2010).

[4] P.K. Ghosh, F. Marchesoni, S.E. Savel'ev, F. Nori, Phys. Rev. Lett. 104, 020601 (2010); P. K. Ghosh, P. Hänggi, F. Marchesoni, F. Nori,and G. Schmid, Phys. Rev. E 86, 021112 (2012); P. Hänggi, F. Marchesoni, S. Savelev, and G. Schmid, Phys. Rev. E 82, 041121 (2010).

[5] M. H. Jacobs, Diffusion Processes (Springer, New York, 1967); R. Zwanzig, J. Phys. Chem. 96, 3926 (1992).

[6] D. Reguera, G. Schmid, P. S. Burada, J. M. Rubi, P. Reimann, and P. Hänggi, Phys. Rev. Lett. 96, 130603 (2006); D. Reguera, A. Luque, P. S. Burada, G. Schmid, J. M. Rubi, and P. Hänggi, Phys. Rev. Lett. 108, 020604 (2012); S. Martens, A. V. Straube, G. Schmid, L. Schimansky-Geier, and P. Hänggi, Phys. Rev. Lett.110, 010601 (2013).

[7] P. Kalinay, Phys. Rev. E 89, 042123 (2014); P. Kalinay, Phys. Rev. E 87, 032143 (2013); P. Kalinay and J. K. Percus, Phys. Rev. E 74, 041203 (2006).

[8] S. Martens, G. Schmid, L. Schimansky-Geier, and P. Hänggi, Phys. Rev. E 83, 051135(2011);N. Laachi, M. Kenward, E.Yariv, and K. D. Dorfman, EPL 80, 50009 (2007).

[9] M. Das and D. S. Ray, Phys. Rev. E 88, 032122 (2013); M. Das, D. Mondal, and D. S. Ray, J. Chem. Phys. 136, 114104 (2012); M. Das, Phys. Rev. E 89, 032130 (2014).

[10] B. Q. Ai and L. G. Liu, Phys. Rev. E 74, 051114 (2006); B. Q. Ai, Phys. Rev. E 80, 011113 (2009).

[11] I. Pineda, J. Alvarez-Ramirez, and L. Dagdug, J. Chem. Phys. 137, 174103 (2012); L. Dagdug and I. Pineda, J. Chem. Phys. 137, 024107 (2012). 
[12] B. Q. Ai, Q. Y. Chen, Y. F. He, F. G. Li, and W. R. Zhong, Phys. Rev. E 88, 062129 (2013).

[13] R. Großmann, L. Schimansky-Geier, and P. Romanczuk, New J. Phys. 14, 073033 (2012).

[14] J. Tailleur and M. E. Cates, Phys. Rev. Lett. 100, 218103 (2008).

[15] Y. Fily and M. C. Marchetti, Phys. Rev. Lett. 108, 235702 (2012).

[16] A. Kaiser, K. Popowa, H. H. Wensink, and H. Löwen, Phys. Rev. E 88, 022311 (2013).

[17] T. Bickel, A. Majee, and A. Wurger, Phys. Rev. E 88, 012301 (2013).

[18] I. Buttinoni, J. Bialke, F. Kummel, H. Löwen, C. Bechinger, and T. Speck, Phys. Rev. Lett. 110, 238301 (2013); F. Kummel, B. ten Hagen, R. Wittkowski, I. Buttinoni, R. Eichhorn, G. Volpe, H. Löwen, and C. Bechinger, Phys. Rev. Lett. 110, 198302 (2013).

[19] S. Mishra, K. Tunstrom, I. D. Couzin, and C. Huepe, Phys. Rev. E 86, 011901 (2012).

[20] T. Ohta and T. Ohkuma, Phys. Rev. Lett. 102, 154101 (2009).

[21] F. Peruani, T. Klauss, A. Deutsch, and A. Voss-Boehme, Phys. Rev. Lett. 106, 128101 (2011).

[22] A. Czirók, A. L. Barabási, and T. Vicsek, Phys. Rev. Lett. 82, 209 (1999).

[23] M. Enculescu and H. Stark, Phys. Rev. Lett. 107, 058301 (2011).

[24] C. Weber, P. K. Radtke, L. Schimansky-Geier, and P. Hänggi, Phys. Rev. E 84, 011132 (2011).

[25] L. Angelani, R. Di Leonardo, and G. Ruocco, Phys. Rev. Lett. 102, 048104 (2009).

[26] M. B. Wan, C. J. Olson Reichhardt, Z. Nussinov, and C. Reichhardt, Phys. Rev. Lett. 101, $018102(2008)$.

[27] P. K. Ghosh, V. R. Misko, F. Marchesoni, and F. Nori, Phys. Rev. Lett. 110, 268301 (2013).

[28] A. Pototsky, A. M. Hahn, and H. Stark, Phys. Rev. E 87, 042124 (2013).

[29] P. K. Ghosh, P. Hänggi, F. Marchesoni, and F. Nori, Phys. Rev. E 89, 062115 (2014).

[30] P. K. Ghosh, J. Chem. Phys. 141, 061102 (2014).

[31] E. Yariv and O. Schnitzer, Phys. Rev. E 90, 032115 (2014).

[32] Z. Csahok, F. Family, and T. Vicsek, Phys. Rev. E 55, 5179 (1997); A. Igarashi, S. Tsukamoto, and H. Goko, Phys. Rev. E 64, 051908 (2001); S. Savelev, F. Marchesoni, and F. Nori, Phys. Rev. E 71, 011107 (2005).

[33] D. Dan, M. C. Mahato, and A. M. Jayannavar, Phys. Rev. E. 63, 056307 (2001); B. Q. Ai, Phys. Rev. E 80, 011113 (2009). 